\title{
Theory of relaxation oscillations in semiconductor quantum dot lasers
}

Cite as: Appl. Phys. Lett. 89, 101107 (2006); https://doi.org/10.1063/1.2346224

Submitted: 16 January 2006 • Accepted: 25 July 2006 Published Online: 06 September 2006

Ermin Malić, Kwang J. Ahn, Moritz J. P. Bormann, et al.

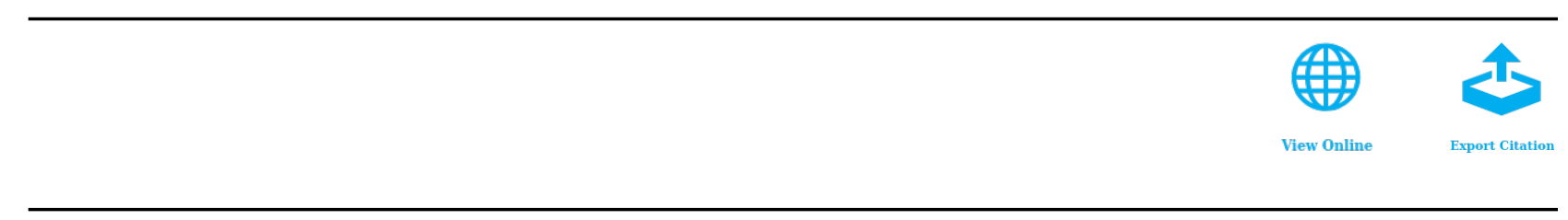

\section{ARTICLES YOU MAY BE INTERESTED IN}

Perspective: The future of quantum dot photonic integrated circuits

APL Photonics 3, 030901 (2018); https://doi.org/10.1063/1.5021345

Tutorial on narrow linewidth tunable semiconductor lasers using Si/III-V heterogeneous integration

APL Photonics 4, 111101 (2019); https://doi.org/10.1063/1.5124254

Multidimensional quantum well laser and temperature dependence of its threshold current Applied Physics Letters 40, 939 (1982); https://doi.org/10.1063/1.92959

母QBLOX

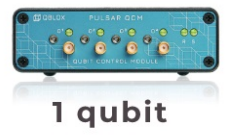

Shorten Setup Time Auto-Calibration More Qubits

Fully-integrated Quantum Control Stacks Ultrastable DC to $18.5 \mathrm{GHz}$ Synchronized $<<1$ ns Ultralow noise

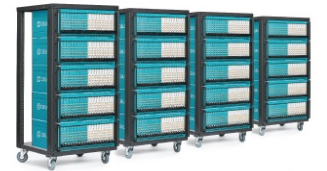

100s qubits

visit our website > 


\title{
Theory of relaxation oscillations in semiconductor quantum dot lasers
}

\author{
Ermin Malić, ${ }^{\text {a) }}$ Kwang J. Ahn, Moritz J. P. Bormann, Philipp Hövel, ${ }^{\text {b) }}$ \\ Eckehard Schöll, and Andreas Knorr \\ Institut für Theoretische Physik, Technische Universität Berlin, 10623 Berlin, Germany \\ Matthias Kuntz and Dieter Bimberg \\ Institut für Festkörperphysik, Technische Universität Berlin, 10623 Berlin, Germany \\ and Center of Nanophotonics, Technische Universität Berlin, 10623 Berlin, Germany
}

(Received 16 January 2006; accepted 25 July 2006; published online 6 September 2006)

\begin{abstract}
A microscopic approach combining rate equations for photon and electron/hole occupations with kinetic equations for Coulomb scattering rates involving quantum dot and wetting layer states in InAs/GaAs quantum dot lasers is presented. The authors find strong damping of relaxation oscillations on a picosecond to nanosecond time scale depending on the type of the initial perturbation, similar to the damping observed in experiments by various groups. They show that the Coulomb interaction is crucial for an understanding of this characteristic strong damping. (C) 2006 American Institute of Physics. [DOI: 10.1063/1.2346224]
\end{abstract}

Currently, there is an increasing interest in developing infrared quantum dot (QD) lasers for high-speed data transmission. Transmission with a low bit-error rate (BER) of 10-12 (Refs. 1 and 2) and eye patterns with a large temperature stability between 20 and $70{ }^{\circ} \mathrm{C}$ (Ref. 3) have been demonstrated at $10 \mathrm{Gbits} / \mathrm{s}$ and $1.3 \mathrm{~m}$ emission wavelength. Large cutoff frequencies of $12 \mathrm{GHz}$ with vertically coupled QDs (Ref. 4) and of $24 \mathrm{GHz}$ based on tunnel injection of carriers into the QDs (Ref. 5) show the feasibility of even higher data transmission rates. Since the modulation bandwidth is strongly dependent on the frequency and damping of the relaxation oscillations (ROs), a detailed theoretical investigation of the dynamics of QD lasers is necessary to optimize these devices. In particular, QD lasers have been found to show a stronger damping of the ROs compared to quantum well (QW) lasers. ${ }^{6,7}$ Damped ROs are known to be advantageous for open eyes and low BER at large bit rates. The underlying dynamic mechanisms are still being discussed. ${ }^{8,9}$

In this letter, a theoretical analysis of the ultrafast RO dynamics of single-mode InAs/GaAs quantum dot lasers is presented. We go beyond standard phenomenological laser rate equations ${ }^{10-14}$ by incorporating microscopic kinetic equations which describe Coulomb scattering processes. ${ }^{15}$ This approach takes into account the microscopic interaction and charge transfer between QDs and the wetting layer (WL) evolving in the process of fabricating self-organized QDs. ${ }^{16}$ Electron-phonon scattering is included for the cooling process in the WL, but neglected for scattering into the QD states, since it is dominated by Coulomb scattering in the lasing regime with high WL carrier density. ${ }^{17}$ We show that for typical QD lasers, the interplay of the induced emission and absorption and the scattering processes leads to ROs in the emission intensity. We investigate the laser dynamics (i) after a weak perturbation, e.g., after applying an optical femtosecond pulse, ${ }^{18}$ and (ii) during the turn-on processes of an InAs/GaAs QD laser pumped by a nanosecond current pulse. ${ }^{19}$ The resulting ROs are compared with recent experimental results.

\footnotetext{
${ }^{a}$ Electronic mail: ermin@itp.physik.tu-berlin.de

${ }^{b}$ Electronic mail: phoevel@physik.tu-berlin.de
}

Since the carrier relaxation processes within the WL and within the QD states (at high WL density ${ }^{20}$ ) are much faster (approximately picoseconds) than capture processes, only the energetically lowest electron $(e)$ and hole $(h)$ levels in the QDs contribute crucially to the laser dynamics. ${ }^{15}$ We use microscopically calculated scattering rates for all relevant processes occurring in the set of coupled nonlinear rate equations for the photon density $n_{\mathrm{ph}}$ and the charge carrier densities $n_{b}$ in the QDs (where $b=e$ and $h$ ),

$$
\begin{aligned}
& \dot{n}_{b}=-\frac{1}{\tau_{b}} n_{b}+S_{b}^{\mathrm{in}} N^{\mathrm{QD}}-R_{\mathrm{ind}}\left(n_{e}, n_{h}\right)-R_{\mathrm{sp}}\left(n_{e}, n_{h}\right), \\
& \dot{n}_{\mathrm{ph}}=-2 \kappa n_{\mathrm{ph}}+\Gamma R_{\mathrm{ind}}\left(n_{e}, n_{h}\right)+\beta R_{\mathrm{sp}}\left(n_{e}, n_{h}\right) .
\end{aligned}
$$

Here, the Coulomb scattering rates $\tau_{e l h}^{-1}=S_{e / h}^{\text {in }}+S_{e / h}^{\text {out }}$ have to be determined from a Boltzmann-like equation for $n_{e}$ and $n_{h}{ }^{15}$ $\Gamma, \kappa, N^{\mathrm{QD}}$, and $\beta$ are the optical confinement factor, ${ }^{21}$ the cavity loss, the QD density, and the spontaneous emission coefficient, respectively. $R_{\text {ind }}:=\hat{W}\left(n_{e}+n_{h}-N^{\mathrm{QD}}\right) n_{\mathrm{ph}}$ is the induced emission rate whereas $R_{\mathrm{sp}}:=\widetilde{W} n_{e} n_{h}$ is the spontaneous emission rate governed by bimolecular recombination. ${ }^{11,12}$ Both spontaneous emission and induced processes are proportional to the Einstein coefficient $W$ (where $\hat{W}:=W A$ with the normalization area $A$ and $\left.\widetilde{W}:=W / N^{\mathrm{QD}}\right)$. The carrier-light interaction is considered with the assumption of only one effective light mode (single photon number $n_{\text {ph }}$ ) dominating over all other modes. The scattering rates $S_{e}^{\text {in }}, S_{e}^{\text {out }}, S_{h}^{\text {in }}$, and $S_{h}^{\text {out }}$ for electrons and holes are calculated microscopically as a function of the WL electron/hole density $w_{\text {elh }}$; the Coulomb interaction is considered up to the second order in the screened Coulomb potential and under the restriction to time scales where the Markov approximation is applicable, ${ }^{22,23}$

$$
\dot{\rho}_{b}=S_{b}^{\text {in }}\left(1-\rho_{b}\right)-S_{b}^{\text {out }} \rho_{b},
$$

where $\rho_{b}$ is the occupation probability in the electron/hole QD state $(b=e$ and $h)$. This Boltzmann-like equation contains Coulomb scattering rates given by 


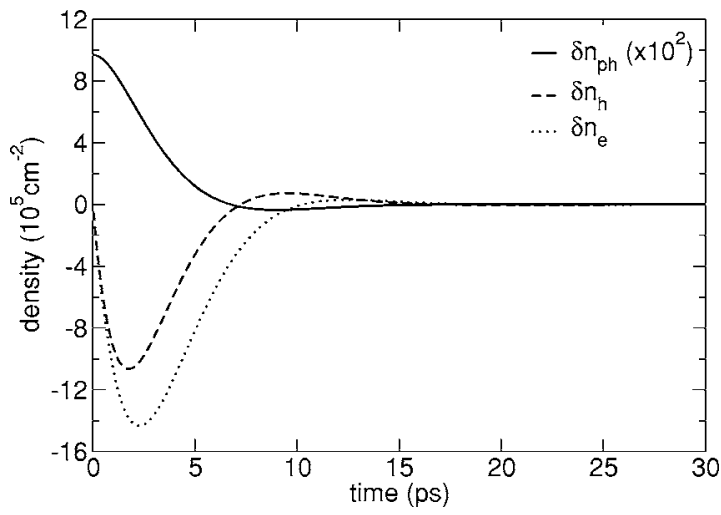

FIG. 1. Time evolution of the deviations of the photon density $\delta n_{\mathrm{ph}}$ and of the QD electron/hole densities $\delta n_{e / h}$ from their steady state $n_{\mathrm{ph}}^{\mathrm{st}}, n_{e}^{\mathrm{st}}, n_{h}^{\mathrm{st}}$ for fixed WL carrier density $w_{e / h}=8 \times 10^{12} \mathrm{~cm}^{-2}$ and for the initial values $\delta n_{\mathrm{ph}}(0)=0.01 n_{\mathrm{ph}}^{\mathrm{st}}=9.7 \times 10^{3} \mathrm{~cm}^{-2}$ and $\delta n_{e}(0)=\delta n_{h}(0)=0$.

$$
\begin{aligned}
S_{b}^{\mathrm{in} / \mathrm{out}}= & \frac{2 \pi}{\hbar} \sum_{l m n} M_{b n l m}\left(M_{b n l m}^{*}-M_{b n m l}^{*}\right) f_{l m n}^{\mathrm{in} / \mathrm{out}} \\
& \times \delta\left(E_{b}+E_{n}-E_{l}-E_{m}\right),
\end{aligned}
$$

with the sum over all WL states (occupation $\rho_{l}, \rho_{m}$, and $\rho_{n}$ ) and $f_{l m n}^{\text {in }}=\rho_{l} \rho_{m}\left(1-\rho_{n}\right)$ and $f_{l m n}^{\text {out }}=\rho_{n}\left(1-\rho_{m}\right)\left(1-\rho_{l}\right) . E_{b}, E_{l}, E_{m}$, and $E_{n}$ are the respective energies. For the evaluation of the Coulomb matrix elements $M_{a b c d}$ and the scattering integrals, we use the approach of Refs. 7 and 12: for a WL extended in the $x-y$ plane, the wave function for the whole QD-WL system $\Phi_{l, \sigma}^{b}(\mathbf{r})=\varphi_{l}^{b}(\boldsymbol{\rho}) \xi_{\sigma}^{b}(z) u^{b}(\mathbf{r})$ can be separated into the inplane component $\varphi_{l}^{b}(\boldsymbol{\rho})$, the $z$ component $\xi_{\sigma}^{b}(z)$, and the Bloch function $u^{b}(\mathbf{r})$. The in-plane component of the confined QD states is approximated by the eigenfunctions of the two-dimensional harmonic oscillator. ${ }^{24}$ The in-plane component of the WL states is approximated by orthogonalized plane waves. Strong confinement in the direction perpendicular to the WL is considered by an infinite barrier using the effective well width approximation. ${ }^{25}$ The calculation of the scattering rates takes the quasi-Fermi distribution over the involved electronic states into account. Furthermore, we include screening by applying the two-dimensional static limit of the dynamic Lindhard equation as well as a background dielectric constant $\varepsilon_{\text {bg }}$ (for details see Ref. 15).

The parameters ${ }^{26}$ needed for the evaluation of Eqs. (1) and (2) are fixed in agreement with recent experimentally investigated QD laser structures. 1,27

First, we focus on the response of a running laser to a weak deltalike perturbation in time $(t=0)$, such as the application of a femtosecond optical pulse. ${ }^{18,28}$ Figure 1 shows the dynamics of the deviations $\delta n_{e}, \delta n_{h}$, and $\delta n_{\mathrm{ph}}$ of the QD electron, hole, and photon densities from their respective stationary values, as a response to a weak perturbation. A single strongly damped RO on a picosecond time scale appears for all three quantities. Note that the different scattering times for holes and electrons lead to a slightly different dynamics of $n_{e}$ and $n_{h}$. The overdamping of the oscillations results from rather large scattering rates (inverse picosecond range) under the considered laser conditions. After less than 20 ps the steady state values are reached. To demonstrate the significance of the scattering rates more clearly, Fig. 2 shows the ROs obtained for a tenfold artificial reduction compared to the original values used in Fig. 1. Now, a damped but pronounced RO emerges. Note that for large perturbations, a

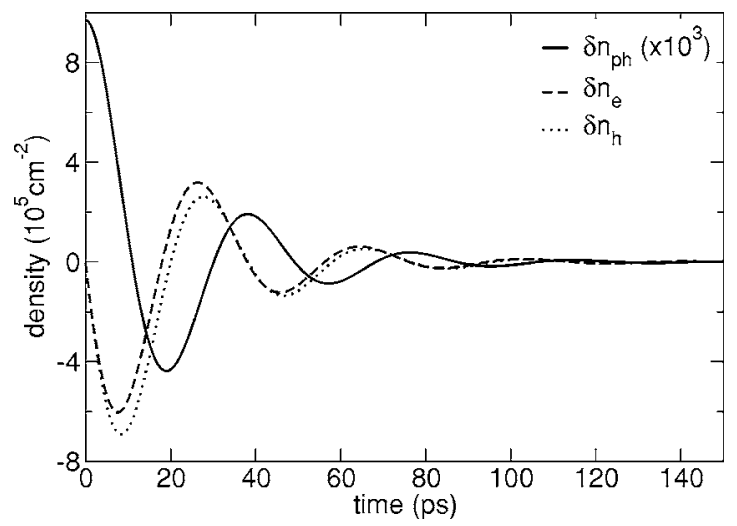

FIG. 2. Relaxation oscillations for artificially reduced scattering rates (tentimes). All other parameters are as in Fig. 1.

small signal analysis (linearization around the steady state) would produce physically unacceptable negative values of the photon density $n_{\mathrm{ph}}$. In that case, nonlinear terms slow down the induced processes resulting in a positive photon density. Depending on initial values, the nonlinear contributions can also lead to an amplification of the deviation from the stationary values.

As second application, the current pulse turn-on dynamics of a QD laser is discussed. ${ }^{19}$ To obtain an inversion an injection current density pulse $j(t)$ of 5 ns width (symmetric Gaussian-like pulse with a rise and fall time of $100 \mathrm{ps)}$ is applied to the noninverted system. During the pulse, the WL states are filled with charge carriers. As a result, the probability of scattering processes between the WL and the QD states varies in time. Hence, the WL electron and hole densities cannot be assumed as constant. Therefore, the system of differential equations [Eqs. (1) and (2)] is extended by two rate equations describing the dynamics of the WL electron and hole densities $w_{b}$ (where $b=e$ and $h$ ),

$$
\dot{w}_{b}=\frac{j(t)}{e_{o}}+\frac{c}{\tau_{b}} n_{b}-S_{b}^{\mathrm{in}} N^{\mathrm{WL}}-\widetilde{R}_{\mathrm{sp}}\left(w_{e}, w_{h}\right),
$$

with the elementary charge $e_{0}$ and with $c=N^{\mathrm{WL}} / N^{\mathrm{QD}}$ where $N^{\mathrm{WL}}=4 \times 10^{13} \mathrm{~cm}^{-2}$ is the WL effective density of states. Moreover, the WL spontaneous recombination rate is given by $\tilde{R}_{\text {sp }}\left(w_{e}, w_{h}\right)=\tilde{W}^{\prime} w_{e} w_{h}$ with $\tilde{W}^{\prime}=\tilde{W} / c$. The strongly nonlinear dependence of the scattering rates on $w_{e}$ and $w_{h}$ is taken into account explicitly. The peak current density is assumed to be closely above the laser threshold. The numerical integration of the complete system of nonlinear differential equations [Eqs. (1), (2), and (5)] is shown in Fig. 3. After a turn-on delay of $0.8 \mathrm{~ns}$, the photon density $n_{\mathrm{ph}}$ increases exponentially. It performs relaxations oscillations with a frequency of $12.5 \mathrm{GHz}$ that are damped towards a stationary value. These oscillations result from the interplay of electron filling, induced emission, and absorption processes. The delay time is due to the initially empty QD states that first need to be filled with charge carriers. After the current pulse is switched off, $n_{\mathrm{ph}}, n_{e}$, and $n_{h}$ decay exponentially. The dynamics of carrier densities shows less pronounced oscillations due to the capacitative inertia of charge carriers. The rise time is dominated by the in-scattering term (picosecond range) in Eq. (1), whereas the fall time is much longer due to slower radiative processes (nanosecond range). The electron density $n_{e}$ has a higher stationary value than $n_{h}$ due to the larger electron in-scattering rate $S_{e}^{\text {in }}$. In Fig. 3(c), the results 


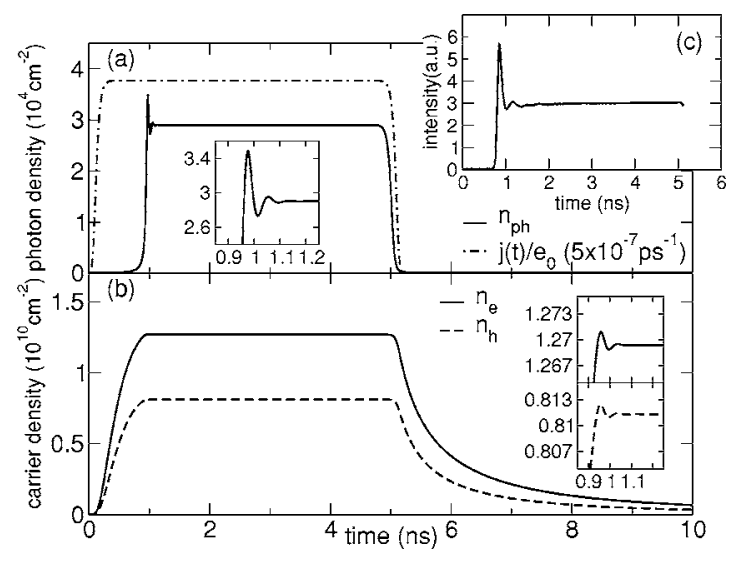

FIG. 3. Response of (a) photon density $n_{\mathrm{ph}}(t)$ and (b) QD carrier densities $n_{e}(t), n_{h}(t)$ to a current pulse (dashed) $j(t)=j_{0} \exp \left[-\left(\left(t-t_{0}\right) / 2.5 \mathrm{~ns}\right)^{56}\right]$ with $j_{0}=1.1 j_{t}$ where $j_{t}$ is the threshold current density. $n_{\mathrm{ph}}(0)=n_{b}(0)=0, w_{b}(0)$ $=10^{-3} w_{b}^{\max }, b=e$ and $h$. Insets: blowups of ROs. (c) Experimental result for InAs/GaAs QD laser with $1.3 \mu \mathrm{m}$ wavelength (Ref. 27).

are compared to the ROs measured in QD lasers that correspond to our modeled WL-QD structure. ${ }^{27}$ In the experiment, the photon density shows dynamics that is in reasonable agreement with the theoretical result. In both cases, similar pronounced ROs with a frequency on an inverse nanosecond time scale are obtained. In particular, the time delay of the dynamics is reproduced ( $0.8 \mathrm{~ns})$, the oscillation frequency, however, is predicted to be twice as fast as in the experiment. This deviation may probably be traced back to several approximations, such as the use of a single mode theory and the neglect of WL-bulk interactions that could slow down the dynamics.

In conclusion, an approach combining rate equations with microscopic kinetic equations has been presented and explains the experimentally found strong damping of ROs in QD lasers dominated by Coulomb scattering rates. Qualitatively similar results are expected if direct bulk-QD interactions are considered.

The authors thank H. C. Schneider (Kaiserslautern), M. Lorke, and F. Jahnke (Bremen) for valuable discussions. One of the authors (E.M.) is grateful to Friedrich-Ebert Stiftung, Studienstiftung des Deutschen Volkes, and Wilhelm-LenzeStiftung for financial support. This work was supported by the DFG through Sfb 296.
${ }^{1}$ M. Kuntz, G. Fiol, M. Lammlin, C. Schubert, A. R. Kovsh, A. Jacob, A. Umbach, and D. Bimberg, Electron. Lett. 41, 244 (2005).

${ }^{2}$ K. T. Tan, C. Marinelli, M. G. Thompson, A. Wonfor, M. Silver, R. Sellin, R. Penty, I. H. White, M. Kuntz, M. Lammlin, N. N. Ledentsov, D. Bimberg, A. E. Zhukov, V. M. Ustinov, and A. R. Kovsh, IEEE Photonics Technol. Lett. 16, 1415 (2004).

${ }^{3}$ K. Otsubo, N. Hatori, M. Ishida, S. Okumura, T. Akiyama, Y. Nakata, H. Ebe, M. Sugawara, and Y. Arakawa, Jpn. J. Appl. Phys., Part 2 43, L1124 (2004).

${ }^{4}$ S. M. Kim, Y. Wang, M. Keever, and J. S. Harris, IEEE Photonics Technol. Lett. 16, 377 (2004).

${ }^{5}$ Z. Mi, P. Bhattacharya, and S. Fathpour, Appl. Phys. Lett. 86, 153109 (2005).

${ }^{6}$ H.-J. Klein, D. Bimberg, H. Beneking, J. Kuhl, and E. O. Gobel, Appl. Phys. Lett. 41, 394 (1982).

${ }^{7}$ D. Bimberg, K. Ketterer, E. Böttcher, and E. Schöll, Int. J. Electron. 60, 23 (1986).

${ }^{8}$ D. G. Deppe, H. Huang, and O. B. D. Shchekin, IEEE J. Quantum Electron. 38, 1587 (2002).

${ }^{9}$ W. W. Chow and S. W. Koch, IEEE J. Quantum Electron. 41, 495 (2005).

${ }^{10}$ H. Haken, Light (North-Holland, Amsterdam, 1985), Vol. 2.

${ }^{11}$ E. Schöll, D. Bimberg, H. Schumacher, and P. Landsberg, IEEE J. Quantum Electron. 20, 394 (1984).

${ }^{12}$ E. Schöll, IEEE J. Quantum Electron. 24, 435 (1988).

${ }^{13}$ W. W. Chow, S. W. Koch, and M. Sargent, Semiconductor Laser Physics (Springer, Berlin, 1994).

${ }^{14}$ D. O'Brien, S. Hegarty, G. Huyet, and A. V. Uskov, Opt. Lett. 29, 1072 (2004).

${ }^{15}$ T. R. Nielsen, P. Gartner, and F. Jahnke, Phys. Rev. B 69, 235314 (2004).

${ }^{16}$ H. C. Schneider, W. W. Chow, and S. Koch, Phys. Status Solidi B 238, 589 (2003).

${ }^{17}$ M. Lorke, T. R. Nielsen, J. Seebeck, P. Gartner, and F. Jahnke, Phys. Rev. B 73, 085324 (2006).

${ }^{18}$ S. G. Hense and M. Wegener, Phys. Rev. B 55, 9255 (1997).

${ }^{19}$ M. Kuntz, N. N. Ledentsov, D. Bimberg, A. R. Kovsh, V. M. Ustinov, A. E. Zhukov, and Yu. M. Shernyakov, Appl. Phys. Lett. 81, 3846 (2002).

${ }^{20}$ R. Wetzler, A. Wacker, and E. Schöll, J. Appl. Phys. 95, 7966 (2004).

${ }^{21}$ D. Bimberg, M. Grundmann, and N. Ledentsov, Quantum Dot Heterostructures (Wiley, New York, 1999).

${ }^{22}$ M. Lindberg and S. W. Koch, Phys. Rev. B 38, 3342 (1988).

${ }^{23}$ F. Rossi and T. Kuhn, Rev. Mod. Phys. 74, 895 (2002).

${ }^{24}$ A. Wojs, P. Hawrylak, S. Fafard, and L. Jacak, Phys. Rev. B 54, 5604 (1996).

${ }^{25}$ A. Liu, Phys. Rev. B 50, 8569 (1994).

${ }^{26} \kappa=0.4 \mathrm{ps}^{-1}, W=4.7 \times 10^{-3} \mathrm{ps}^{-1}, N^{\mathrm{QD}}=2.0 \times 10^{10} \mathrm{~cm}^{-2}, A=4.0 \times 10^{-9} \mathrm{~m}^{2}$, $\beta=5.0 \times 10^{-5}$, and $\Gamma=5 \times 10^{-3}$. The scattering rates were calculated using Eq. (3) for a WL carrier density of $w_{e}=w_{h}=8 \times 10^{12} \mathrm{~cm}^{-2}: S_{e}^{\text {in }}$ $=0.631 \mathrm{ps}^{-1}, S_{e}^{\text {out }}=0.05 \mathrm{ps}^{-1}, S_{h}^{\text {in }}=0.029 \mathrm{ps}^{-1}, S_{h}^{\text {out }}=0.310 \mathrm{ps}^{-1}$, and $\varepsilon_{\mathrm{og}}$ $=13.18$.

${ }^{27}$ M. Kuntz (unpublished), measured with the QD structure described in Ref. 19.

${ }^{28}$ M. Kira, F. Jahnke, and S. W. Koch, Solid State Commun. 102, 703 (1997). 